
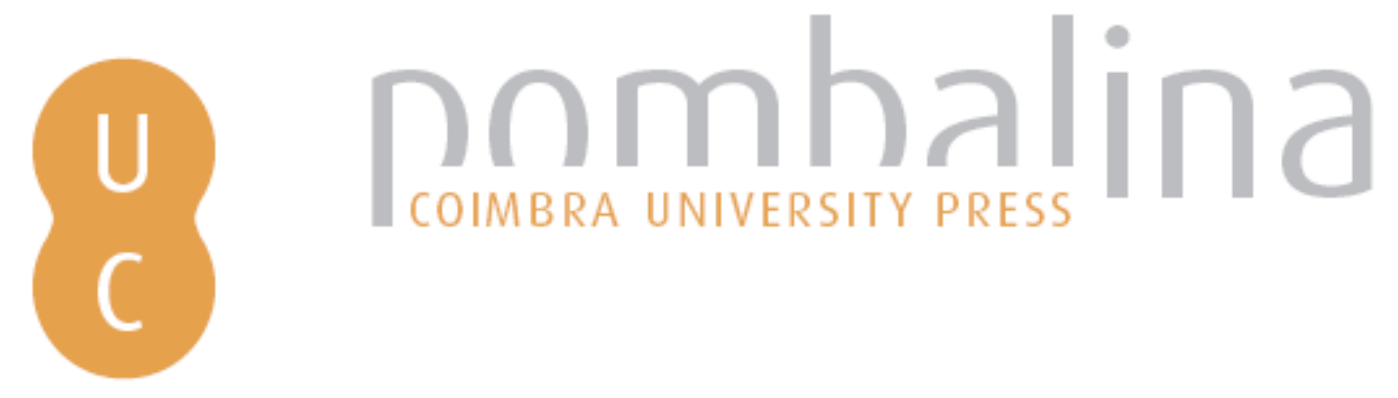

\title{
La enseñanza de las matemáticas en la nueva España: comparación entre la real y pontificia Universidad y el Colegio de Minería
}

\author{
Autor(es): $\quad$ Lazo, Yolanda; Mendoza, Martha \\ Publicado por: Imprensa da Universidade de Coimbra \\ URL \\ persistente: URI:http://hdl.handle.net/10316.2/32402 \\ DOI: $\quad$ DOI:http://dx.doi.org/10.14195/978-989-26-0469-5_17 \\ Accessed : $\quad$ 26-Apr-2023 12:05:25
}

A navegação consulta e descarregamento dos títulos inseridos nas Bibliotecas Digitais UC Digitalis, UC Pombalina e UC Impactum, pressupõem a aceitação plena e sem reservas dos Termos e Condições de Uso destas Bibliotecas Digitais, disponíveis em https://digitalis.uc.pt/pt-pt/termos.

Conforme exposto nos referidos Termos e Condições de Uso, o descarregamento de títulos de acesso restrito requer uma licença válida de autorização devendo o utilizador aceder ao(s) documento(s) a partir de um endereço de IP da instituição detentora da supramencionada licença.

Ao utilizador é apenas permitido o descarregamento para uso pessoal, pelo que o emprego do(s) título(s) descarregado(s) para outro fim, designadamente comercial, carece de autorização do respetivo autor ou editor da obra.

Na medida em que todas as obras da UC Digitalis se encontram protegidas pelo Código do Direito de Autor e Direitos Conexos e demais legislação aplicável, toda a cópia, parcial ou total, deste documento, nos casos em que é legalmente admitida, deverá conter ou fazer-se acompanhar por este aviso.

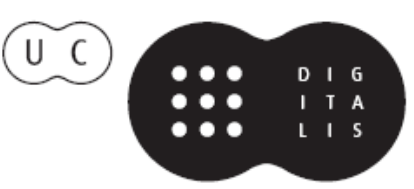


Ana Leunar Pereira João Rui Pita

[ Coordenaçä̃ ]
Rotas da Natureza

Cientistas

Viagens

Expedifgũes

Instituip̧ües

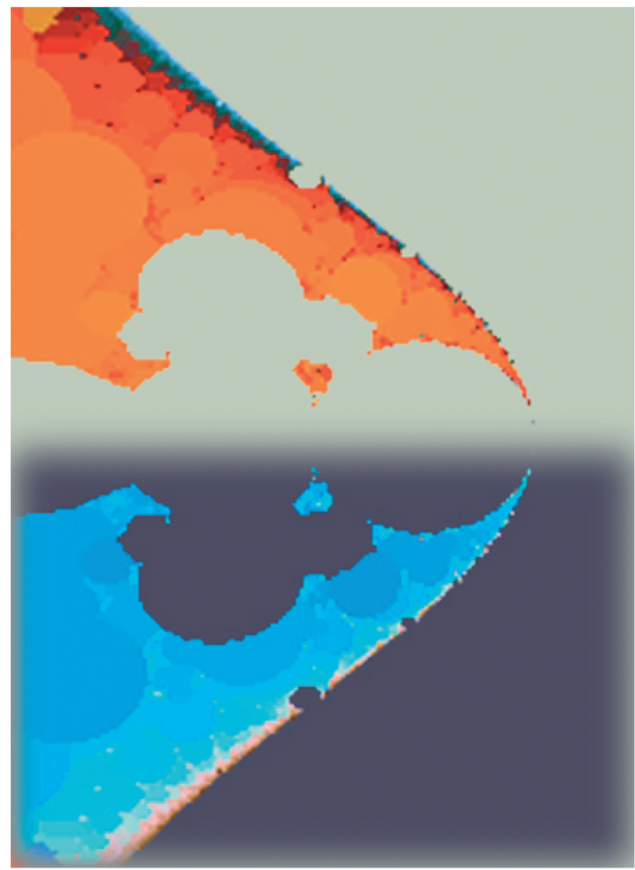




\section{Coordenaçáo Científica da Colecção Ciências e Culturas}

João Rui Pita e Ana Leonor Pereira

Os originais enviados são sujeitos a apreciação científica por referees

\section{Coordenação Editorial}

Maria João Padez Ferreira de Castro

\section{Edição}

Imprensa da Universidade de Coimbra

Email:impresauc@ci.uc.pt

URL: http://www.imp.uc.pt • Normas de publicação de colecçôes

\section{Design}

António Barros

Pré-Impressáo

António Resende

Imprensa da Universidade de Coimbra

\section{Capa}

António Barros, com imagem de E. M. de Melo e Castro, 2003 [Fractal original gerado no Fractint com tratamento no Photoshop 7.0]; Cortesia: António Barros

Impressão e Acabamento

SerSilito • Maia

\section{ISBN}

978-989-8074-12-6

\section{Depósito Legal}

Obra publicada com a colaboraçáo de:
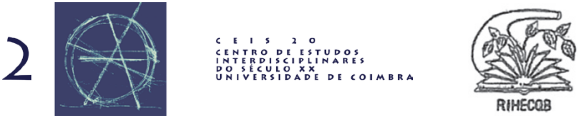

Obra publicada com o apoio de:

FCT Fundação para a Ciência e a Tecnologia

MINISTÉRIO DA CIÊNCIA, TECNOLOGIA E ENSINO SUPERTOR Portug

Programa Operacional Ciência, Tecnologia, Inovação do Quadro Comunitário de Apoio III
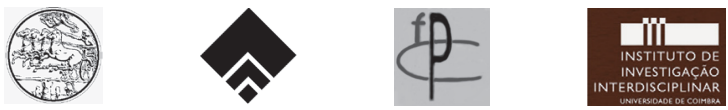

\section{Baxter}

(c) 2006, Imprensa da Universidade de Coimbra 
João Rui Pita

Ana Leonar Pereira

(Courdenação)

Rotas da Natureza

Cientistas

Viagens

Expediçũes

Instituiç̃̃es

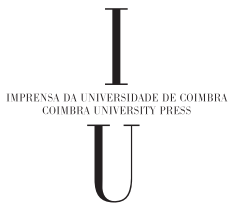

- colmbra 2006 
(Página deixada propositadamente em branco) 
Yolanda Lazo e Martha Mendoza

Universidad Autónoma Metropolitana, Facultad de Filosofía y Letras, UNAM, México

\section{LA ENSEŃANZA DE LAS MATEMÁTICAS EN LA NUEVA ESPAÑa: COMPARACIÓN ENTRE LA REAL Y PONTIFICIA UNIVERSIDAD Y EL COLEGIO DE MINERÍA}

La cátedra de astrología y matemáticas de la Real y Pontificia Universidad de México se privilegió de haber contado con profesores quienes no permanecieron al margen de las diferentes corrientes provenientes de las reformas borbónicas como protagonistas de este cambio mencionaremos a diversos criollos novo hispanos, quienes se desenvolvieron tanto dentro del espacio universitario como en los campos de la astronomía. Lo anterior se exhibe en los libros y manuscritos que llegan a nuestras manos y en el contenido de los exámenes de oposición que sustentaron con el propósito de obtener la titularidad de la cátedra.

El Colegio de Minería abrió sus puertas en el año de 1792, derivación del esfuerzo de uno de los catedráticos de la Real Universidad, la cual llamaríamos hoy una escuela alternativa, con programas y planes de estudio diferentes a los del arraigo oficial y religioso. La Corona apoyo el proyecto, como parte de las innovaciones que les permitían entrar en el mundo de la ilustración defendiendo la supremacía del estado sobre la iglesia, asociado a que los minerales eran elemento clave para la economía del virreinato. Las matemáticas, jugaron un papel central, indispensables para concebir la física y la química, se distinguieron por su ramificación temática, los textos estuvieron a la altura de las universidades europeas. Se contó con laboratorios y modernos instrumentos de medición y de aplicación práctica, consiguiendo ser el primero en América en cuanto a la difusión institucionalizada de la ciencia moderna.

Cinco décadas después de la caída del mas grande imperio de América en 1553 se fundó la Real Universidad. En 1637 a petición de un grupo de alumnos encabezados por Fray Diego Rodríguez lograron la creación de la cátedra de astrología y matemáticas asignatura indispensable para los estudiantes de medicina dado que les proporcionaba las percepciones de la influencia de los astros con las correspondencias de los órganos del cuerpo con los signos del zodiaco. Diego Rodríguez, fue un personaje destacado en el campo de las ciencias exactas, al igual que sucedió con los pensadores del viejo mundo, vivió la coyuntura entre las creencias tradicionales y las de corte moderno de esta manera durante los 31 años que estuvo al frente de la cátedra muy probablemente conoció a de Revolutionibus Orbium Coelestium de Nicolas Copernico, ya que los planes de estudio de la cátedra lo incluían, al igual que algunas universidades europeas, específicamente la Universidad de Salamanca en España; sin embargo es- 
tos estatutos también aconsejaban leer las ortodoxas concepciones del Almagesto de Ptolomeo (siglo II d.c.),

Este personaje calculo las condiciones en que ocurriría un eclipse en 1641 basándose en el novae motuum ephemerides Brand, del alemán David Origanus publicadas 1609 , obra que se apoyo en otras que usaban las tablas astronómicas de Copernico ${ }^{1}$, aunque los movimientos planetarios seguían el modelo de Tycho Brahe, calculo por primera vez, la longitud de la ciudad de México, en 5 horas 45 minutos 50 segundos, o sea 101 grados 27 minutos y 30 segundos al occidente de París. Medición que fue mas precisa que la calculada por el Barón de Humboldt, 165 años después y corregida hasta el siglo XIX. Lego seis obras manuscritas y un impreso, tres de matemáticas, dos de astronomía y una sobre construcción de aparatos científicos.

Carlos de Sigüenza y Góngora (1645-1700), fue una de las figuras científicas sobresalientes del virreinato, profesor durante 22 anos de la cátedra de astrología y matemáticas en la Real y Pontificia Universidad. Su conocido texto La libra astronómica y filosophica, (1690) testifica científicamente en la aparición en la bóveda celeste de un brillante cometa ocurrido entre noviembre de 1680 a marzo de 1681, dichos fenómenos ancestralmente había sido interpretados como mensajeros de nefastos maleficios. Sigüenza en su empeño por proporcionar una versión mas racional de los hechos se hace acreedor al merito de haber sido el primero en expresar el acontecimiento como un fenómeno natural.

El Dr. Marco Antonio Moreno Corral, hace un análisis riguroso del texto explica que desde el punto de vista astronómico es el mas completo publicado en tierras mexicanas durante el siglo XVII, que su lenguaje se estima científico y cuenta con valores de modernidad en los saberes de la ciencia moderna. ${ }^{2}$

La finalidad del escrito fue rebatir aseveraciones del jesuita Eusebio Kino, quien publico un libro Exposición astronómica del Cometa, argumentando que la aparición del cometa provocaría influencias maléficas sobre los seres humanos presagiando calamidades especialmente para los países europeos del norte, conclusión debida a la inclinación del follaje de su cauda.

Añade Moreno Corral, que Don Carlos describe la trayectoria del cometa en la bóveda celeste, basando sus observaciones con un buen sextante fabricado en Bruselas y un telescopio refractor de precisión que contaba con una retícula filar, dispositivo mecánico que le permitió medir desplazamientos angulares muy pequeños del mismo; el manejo apropiado de estos aparatos y el rigor de sus cálculos permitieron establecer con exactitud su trayectoria, teniendo como características principales que su cola tenia 10 grados de longitud, (veinte veces el diámetro lunar), posteriormente el 30 de diciembre alcanzo su máxima extensión con dimensiones de mas de 65 grados, lo que implicaría que cualquier espectador interesado pudo haber visto que la cauda se extendía por mas de un tercio de la bóveda celeste.

Otros datos significativos que consigna con precisión fueron la velocidad, dirección, tipo de movimiento directo y orbita parabólica; además de descubrir que la

\footnotetext{
${ }^{1}$ Moreno Corral, Marco Arturo, Berron Mena, Tannia, .Siguenza y Góngora: un científico de transición Quipu, Revista Latinoamericana de Historia de las ciencias y de la Tecnología, Vol. 13, un. 2, mayo-agosto de 2000, México, D. F., ps 161-173.

2 Ibidem op., cit.
} 
forma de la cauda no fue rectilínea y estuvo constituida por dos colas, esta riqueza de información que Siguenza recopilo, permite hoy en día trazar la trayectoria y dimensiones de este objeto esplendoroso. La observación provoco valiosas discusiones entre personaje de la talla de Halley, Flamsteed y Newton, Ese ultimo en su obra mas importante Philosopia naturalis principia matemática, hace cálculos para determinar la trayectoria de cualquier cometa, concluyendo en un principio que el cometa estudiado por Siguenza presentaba dos colas diferentes, posteriormente al calcular sus orbitas matemáticamente, se percato que se trataba de un solo cuerpo, tal como Siguenza lo había señalado; desafortunadamente el novohispano Siguenza no conoció las teoría newtonianas; no obstante, desde el principio de sus observaciones se percato de que se trataba de un solo cometa. Nuestro personaje puso a disposición de los matemáticos europeos sus datos, desgraciadamente no llegaron oportunamente a Newton, de haber acaecido, con certeza este gran científico las habría aprovechado.

El merito que hoy en día consideraríamos como colateral es que nuestro personaje además de haberle rebatido a Kino, catedrático europeo sus versiones de tipo mágico aristotélicas, demostró científicamente el fenómeno cósmico, sin lugar a dudas hizo buen uso del acervo bibliográfico que poseyó presumía de contar con «la mejor y mayor libreria de aquellas ciencias y esta facultad que jamás ha habido en la Nueva España», en el apéndice de su libro se citan 221 títulos y/o autores, de los cuales 89 son considerados como científicos. Las herramientas matemáticas fueron los Elementos de Euclides aplicando uno de los teoremas para medir las dimensiones de la cauda, refiere el Curso Matemático de Juan Caramuel, a quien menciona como amigo, aplica sus logaritmos, usa de Juan de Zaragoza, La Esfera Celeste.

José Ignacio Bartolache (1739-1790), es considerado otro personaje excepcional, sus publicaciones bastante acreditadas, el Mercurio Volante y el folleto de Las Lecciones Matemáticas, único de una serie frustrada que intentó publicar, en él expone con rigor el método matemático, sigue a Descartes, propone la duda y aconseja ante todo la experimentación. En el transcurso de ocho años, fue profesor de la cátedra de matemáticas y astrología en la Real Universidad, sustituto a Velásquez durante ocho períodos distintos, en su ausencia por el viaje a Baja California, lo suplantó durante dos años,

Otra de las figuras ejemplares en la historia de la ciencia en nuestro país fue, sin duda, Velásquez de León, su grado de actualización se comprueba con la participación que tuvo en 1769 con la expedición de científicos europeos con fin de estudiar el conocido viaje al sur de la península de California del tránsito venusino por el disco solar. Dr. Moreno Corral explica al respecto que los tránsitos venusinos y de mercurio no pueden ser predichos ni explicados correctamente sin basarse en la teoría heliocéntrica. En 1691 Edmund Halley señaló en los Philosophical Transactions publicados por la Royal Society de Londres que los eventos referidos podrían utilizarse para determinar el valor de la paralaje solar, ángulo bajo el que un observador hipotético posicionado en el centro del sol, podría ver el diámetro terrestre, de tal manera que al conocer este valor, sería trivial calcular la distancia absoluta que separa la tierra del sol. ${ }^{3}$

\footnotetext{
${ }^{3}$ Ver: Moreno Corral, Marco Arturo, La Participación mexicana en el estudio de los tránsitos venusinos, Instituto de Astronomía, UNAM, Campus Ensenada, México.
} 
Es importante mencionar que la observación de dicho tránsito requería el ser partidario del modelo heliocéntrico desarrollado por Nicolás Copérnico, que, a su vez, exigía el soporte teórico aportado por Johannes Kepler. Como se anotó esas concepciones y teorías necesariamente tenían que haber sido ampliamente conocidos por todos los observadores del evento referido, inclusive Velásquez de León.

Velásquez en la ciudad de México, fijó con la participación de los matemáticos Ignacio Bartolache y León y Gama, la latitud de la ciudad de México en 19 grados 25 minutos 58 segundos, de latitud norte y $6 \mathrm{~h}$. $46 \mathrm{~min} .55 \mathrm{seg}$. al oeste de Paris; resultando ser la medición más exacta del siglo XVIII, los datos de la longitud fueron 278 grados 15 minutos al Este del meridiano que pasa por la Isla del Fierro. Para ello usó los instrumentos que había heredado de la expedición europea Datos publicados en las Observaciones para determinar la longitud del valle de México.

En 1764, el profesor Juan Gregorio Castro y Martínez renunció a la cátedra de astrología y matemáticas para el caso, la selección se llevaba a cabo mediante un examen de oposición, frente a La Real Junta quien era encargada de asignar los votos a los concursantes, los temas eran seleccionados del libro de la Esfera de Juan de Sacrobosco, el participante contaba con las siguientes 24 horas para preparar el examen que habría de disertar en una hora medido con una ampolleta o reloj de arena, dos de los coopositores contaban con el derecho de cuestionar los argumentos del expositor. ${ }^{4}$

Durante el siglo XVII bastaba la exposición oral como requisito para elegir al expectante profesor, en el siglo XVIII, se estipuló que cada concursante se obligaba a entregar cinco horas después de la disertación verbal, una pequeña tesis escrita con las conclusiones del tema expuesto al jurado. ${ }^{5}$

Joaquín Velásquez de León bajo los reglamentos expuestos, concurso para la titularidad de la cátedra, el tema seccionado al azar fue el asentado en el capítulo siete, del libro uno de la Esfera "terrum esse centrum mundi», o sea «La Tierra como el Centro del Mundo». El texto sostiene que este planeta tiene un figura plana, con dos divisiones, el agua en la parte inferior y el firmamento en la parte superior.

El día 5 de diciembre de 1764, a las ocho de la mañana, Velásquez de León disertó en el Palacio Arzobispal, en presencia del virrey de la Nueva España y de la Real Junta integrada tanto por eclesiásticos de la más alta investidura, como por autoridades superiores de la Universidad Pontificia, quienes aun respetaban el protocolo de estas solemnes ceremonias, mezclando rituales religiosos con administración y selección de profesores.

La traducción de la tesis escrita donde plasmó sus conclusiones es la siguiente:

Aunque moderar a nuestros autores, sobre la inmovilidad de la Tierra y decir es ésta el centro del universo, desde los presagios, casi es evitar a las demás teorías astronómicas y es una teoría incompleta, sin embargo, la siguen los terráqueos, para explicar esta vida, y si nuestro tiempo al estudiar los astros celestes es calculado, y no haciendo decir que es juzgado. Nosotros buscamos, como el sistema del universo, no es tan absoluto al aplicarse por donde se pueda concebir los fenómenos de manera convincente en este escrito creemos con gusto lograr anular hasta aquí y tratar de cam-

\footnotetext{
${ }^{4}$ A.G.N., Constituciones Palafoxianas, constitución LXXIX, f. 95 v., Ramo universidad v 91, f. 585 v.

${ }^{5}$ A.G.N., Universidad, v. 91, f. 585v
} 
biar ese sistema filosófico por el pitagórico, además de restaurar el de Copérnico. El sistema copernicano si acaso se atreve a admitir una hipótesis, que es observacionable y puede ayudar a las teorías astronómicas de una manera perfecta y satisfactoriamente explicar el cosmos. ${ }^{6}$

Seguramente la exposición oral ante un jurado calificado fue acorde con la tesis escrita. Ante lo descrito anteriormente, surgen varios cuestionamientos como los siguientes; ¿sería Velásquez de León un excelente orador?, ¿contaría con una gran capacidad de persuasión?, ¿estarían los integrantes del Claustro Universitario convencidos de las nuevas teorías?, ¿los eclesiásticos aceptaban las nuevas concepciones del universo propagadas por Nicolás Copérnico?, ¿por qué las leyes universitarias conservadoras y obsoletas no se modificaron? En Velásquez de León confluyó otro agravante: era un abogado que sostenía y pretendía convencerlos de las concepciones modernas del universo, cuando la ley estipulaba que fuera medico el profesor de la cátedra.

Posiblemente una de las respuestas -aunque parciales-, sea el hecho que de modificarse los reglamentos de la universidad, al aceptar oficialmente los avances propios de las concepciones de la ciencia moderna, implicaba pérdida de poder para los integrantes del Claustro. Velásquez de León tuvo la visión de crear otra escuela, el Colegio de Minería, posiblemente pensando en la institucionalización de la ciencia moderna, con apoyo de la corona fue nombrado director general, pero lamentablemente muere en la Ciudad de México en 1786.

En 1777, los déspotas ilustrados propiciaron una serie de innovaciones; se creo el Real Colegio Patriótico de Bergara en España, esta mención obedece a que el Colegio de Minería estuvo muy identificado a este instituto, fue copia fiel de su conducción y organización .

Conviene referir como algo de suma importancia que los planes y programas de estudio propuestos por Velásquez de León en 1778 fueron respetados íntegramente por el nuevo director del Real Colegio, Fausto de Elhuyar; no así los elementos de la planta docente, ejemplo de ello es que el matemático León y Gama quien había sido designado por Velásquez de León para impartir las clases de aerometría y pirotecnia, al tomar posesión, sustituyó a los profesores novohispanos por un grupo de peninsulares, de quienes aportaremos detalles de su formación. Fausto de Elhúyar y Zubice, estudió química en París con Rouelle (quien fue profesor de Lavoisier); en compañía de su hermano, se prepararon en metalurgia y métodos de minería en los famosos centros mineros de Europa. Fausto de Elhúyar y Andrés del Río hicieron el aislamiento y descubrimiento del wolframio (tungteno) y del vanadio, nuevos elementos, hechos que les dio un gran prestigio.

El químico Andrés del Río explico la cátedra de mineralogía, estudió cuatro años en París en el College de France, después pasó a Freiberg y a Schemnitz teniendo como condiscípulos a Elhúyar, Humboldt y Linder; su formación la complemento en Inglaterra, Escocia y Francia. En 1797 redactó en español el libro de texto, Elementos de Orictognosia, en 1804 tradujo del alemán las Tablas mineralógicas de Kasrsten.

En 1793 se nombró a Francisco Bataller, exprofesor del Colegio de San Isidro de Madrid para el curso de física, las matemáticas era indispensable haberlas aprobado.

\footnotetext{
${ }^{6}$ La responsabilidad de la traducción es de quien escribe
} 
El libro de texto fue el del astrónomo contemporáneo Sigaund de la Fond, en donde se registraban las teorías de newton, sin embargo, no se contaba con libros de física aplicada a la minería, Elhúyar acordó con Bataller que lo redactara, se tituló Principios de Física Matemática y Experimental, incluyo: propiedades de los cuerpos, mecánica de los sólidos, hidrodinámica y óptica. La clase de química, debería aprenderse después de haber acreditado las matemáticas y la física, el primer curso inició en 1797, teniendo como profesor al director del Seminario, Fausto de Elhuyar. ${ }^{7}$

Desde su apertura la finalidad de la instrucción en el Seminario tuvo una inclinación practica experimental, Se adquirió un gabinete de física bien equipado, con máquinas, hornos e instrumental, además de un laboratorio químico, una colección geológica clasificada según el sistema Werner y otro para minerales, según Humboldt estos hechos dan idea del ardor con que se ha abrazado el estudio de las ciencias exactas en la capital de la Nueva España, al cual se dedican con mucho empeño. ${ }^{8}$

El profesor de matemáticas fue el capitán Andrés José Rodríguez, asignado directamente por Real Orden en 1788, se entiende que había estudiado matemáticas en Madrid y conocía bien las minas de Almaden.

Las diferentes ramas de las matemática fue el objetivo de enseñanza primordial en el colegio, El artículo primero de los estatutos estipula sobre su enseñanza el siguiente plan de estudios:

Primer año: Las matemáticas puras, que comprenderán, aritmética, álgebra, geometría, trigonometría.

Segundo año: Geometría apropiada a las operaciones propias usuales de la minería (incluyendo la geometría subterránea), dinámica hidrodinámica.

Tercer año Chimia reducida al reino animal ( caracteres exteriores y análisis de los minerales) y la metalurgia o tratado de los diversos métodos y operaciones que se benefician generalmente los minerales.

Cuarto año: Física subterránea o teoría de las montanas con introducción al laboreo de las minas, reconocimiento del terreno, excavación subterránea y extracción de los frutos.

Era obligatorio que los futuros peritos facultativos pasaran dos años en las minas próximas a la capital, para que se ejercitaran.

Desde el año 1797, el cálculo diferencial y el integral quedaron junto con la geometría práctica (medidas de las minas) y la trigonometría esférica formando parte del segundo curso de matemáticas, así lo muestran las disertaciones de los actos públicos. En julio1802 se propone al Tribunal incorporarlo institucionalmente.

La bibliografía utilizada en la cátedra de matemáticas fue abundante existen diversos evidencias de su riqueza. En 1799, el profesor y bibliotecario Mariano Fernández de Castro, catalogó los libros de la biblioteca, aunque el inventario fue parcial, por esos rastros sabemos que existieron más de cien títulos diferentes que tratan temas de matemáticas. ${ }^{9}$

\footnotetext{
${ }^{7}$ AHPM ML91B, 1806, f. 193-4 Ver Aceves, Mendoza op. cit. p. 26

${ }^{8}$ AHPM , doc. 1791-III-50-d29, f 38

${ }^{9}$ AHPM caja 100, doc. 23 (1799) catálogo de los libros existentes en la biblioteca, 2 de abril de 1799, elaborado por Mariano Fernández
} 
El director del Colegio considero que el texto más apropiado para la cátedra eran los Elementos de Matemáticas de Benito Bails (1730-1797), editados en España, el permiso para reimprimirlo fue otorgado después de algunas transacciones encubiertas. Entre tanto se adopto como primer texto Los elementos de aritmética, álgebra y geometría de Juan Justo García (1730-1797), complementado con Los Elementos de matemáticas de Lemeaur las Tablas logaritmicas de Gardiner y los indispensables Elementos de Euclides. ${ }^{10}$

El texto es considerado como el trabajo matemático enciclopédico en castellano más importante del siglo XVIII, expone en forma didáctica la teoría con sus últimos adelantos en la física y la astronomía. Introduce por vez primera el calculo infinitesimal y la geometría analítica. Entre los autores que lo fundamentan se encuentran Bernoulli, Cramer, Euclides, Newton, Euler y Lagrange. Los tres primeros tomos estuvieron impresos 1772 , la colección de diez salió al publico en 1779 , esta ultima trata los elementos de aritmética, geometría y trigonometría, álgebra, las cónicas, cálculo infinitesimal y ecuaciones diferenciales; dinámica, hidrodinámica, óptica, elementos de astronomía física, arquitectura civil e hidráulica y tablas de logaritmos. En el primer tomo dedicado a la astronomía, se habla del sistema del mundo y con precauciones se expone a Copérnico. En España sirvió de texto en numerosos centros todos los matemáticos que se formaron en el siglo XVIII lo estudiaron, fue el libro por excelencia del Seminario de Bergara, El Real Seminario de los Nobles, la Escuela de Almadén, la Universidad de Salamanca y en la Academia de San Carlos en la Nueva España. Dada su importancia tuvo una vigencia entre treinta y cuarenta años, fue reeditados en varias ocasiones hasta principios del siglo XIX. En el Colegio de Minería se utilizo hasta 1819. Parece ser que Bails parafrasea en su obra Cours de mathematique a Etienne Bezout (1730-1783), texto que era el equivalente en las universidades francesas. ${ }^{11}$

El análisis expuesto obtenido en fuentes muy diversas tales como manuscritos, tesis, publicaciones periódicas, libros y archivos, permite comentar que la Real y Pontificia Universidad permaneció cerrada a la aceptación de las nuevas teorías. No obstante, como se ha mostrado desde el siglo XVII existieron en la Nueva España profesores de la cátedra de astronomía y matemáticas que mostraron en sus escritos los conocimientos revolucionarios de vanguardia. Posiblemente las autoridades del claustro universitario al poner fin a las concepciones aristotélicas y tolemáticas temían perder su dominio.

El Real Seminario de Minería se convirtió en el instituto de transmisión más importante de la red científica novohispana. El gran merito fue ser pionero en América en la difusión institucionalizada de la ciencia moderna, privilegio que mantuvo por mas de cincuenta anos.

Consiguiendo ser el primero en América en cuanto a la. Liderazgo que habría de mantener en los primeros cincuenta años de vida independiente de la nación mexicana, peculiaridades que permitieron que los gremios inclusive los de la salud asistieran a sus cátedras.

${ }^{10}$ Joaquín, Izquierdo, La primera casa de las ciencias en México, México, 1958, editorial Ciencia, p. 50.

${ }^{11} \mathrm{G}$. Boulingand. A une etape decisive l'álgebra . L’ouvre scientifique d'Etienne Bezout. RGS,55 (1948), 121-123 


\section{Colecçãa \\ 2 Ciências e Culturas Caimbra 2006}

